Reprod. Nutr. Dévelop., 1980, 20 (4 B), 1161-1172.

\title{
Fistulation de l'estomac et contenus gastriques résiduels après ingestion d'un régime semi-purifié à base d'amidon de maïs chez le Porc
}

par J. C. CUBER, J. P. LAPLACE, P. A. VILLIERS

Laboratoire de Physiologie de la Nutrition, I. N. R. A., 78350 jouy en josas

Summary. Fistulation of the stomach and residual gastric contents after intake of a semipurified maize dief in the pig.

Gastric emptying was studied by measuring the residual gastric contents in 6 pigs weighing an average $56.5 \mathrm{~kg}$ and fitted with an indwelling gastric cannula in the middle of the greater curvature of the stomach. The feed offered was a semi-purified test meal, including $1980 \mathrm{~g}$ of fresh matter, $880 \mathrm{~g}$ of dry matter, $61.9 \mathrm{~g}$ of total crude protein $(\mathrm{N} \times 6.25)$, $7.1 \mathrm{~g}$ of soluble crude protein, $720 \mathrm{~g}$ of total starch (glucose and all its $\alpha$-derivatives) and $7 \mathrm{~g}$ of hydrosoluble starch. These components were determined in the gastric contents collected at the following intervals after the meal : $30 \mathrm{~min}, 1 \mathrm{~h}, 2 \mathrm{~h}, 4 \mathrm{~h}$, and $7 \mathrm{~h}$. The inoffensiveness of stomach fistulation was checked by recording the electromyographic activities of the gastric antrum.

The results on gastric emptying are given in figure 2. The total residual amounts of crude protein and starch were closely related to the amounts of dry matter. The soluble crude protein fraction increased stepwise, reaching 55 p. 100 of the total crude protein fraction $7 \mathrm{~h}$ after the meal. The soluble starch fraction was always less than $1 \mathrm{p} .100$ of the total starch fraction. As calculated by difference, the amounts emptied were compared with those measured directly by collecting the duodenal contents in similar experimental conditions. This comparison of emptying kinetics showed large differences according to the technique used. Gastric emptying of dry matter was steadier and more marked within the 7 -hour range when using gastric fistulation. The considerable difference also observed in the case of starch (fig. 3) may be interpreted as an underestimation of duodenal content starch because of the interference of bile pigments with $\mathrm{H}_{2} \mathrm{O}_{2}$ measurement in the enzyme analyses. A comparison of starch emptying kinetics with those of the absorption of reducing sugars suggested that sugar absorption might be restricted due to the velocity of starch hydrolysis by pancreatic $\alpha$-amylase.

\section{Introduction.}

La cinétique d'évacuation gastrique de la matière sèche, de l'amidon et de l'azote, après ingestion d'un régime semi-purifié à base d'amidon de maïs, a été décrife chez le Porc sur la base de collectes réalisées au niveau d'une fistule duodé- 
nale réentrante (Cuber et Laplace, 1979a ef b). Cependant, diverses questions se posent, liées aux effets potentiellement perturbateurs de la fistulation réentrante duodénale (Laplace, 1979) et des manœuvres de collecte ef réintroduction (Low, 1979), aux conséquences des contraintes anatomiques qui amènent à collecter un flux sécrétoire important en plus des effluents gastriques, et à l'interférence du reliquat des repas anférieurs à l'épreuve (Cuber et Laplace, 1979a). En dépit d'une bonne coïncidence avec les données obtenues par la technique des abattages (Rérat et Lougnon, 1963), il nous a paru utile d'évaluer l'évacuation gastrique du même régime par la

mesure directe des quantités résiduelles dans l'estomac chez le Porc équipé d'une canule gastrique permanente.

En effet, la fistulation de l'estomac chez le Porc est une technique utilisée depuis bien plus longlemps que la fistulation réentrante de l'intestin, et qui remonterait à Colin (1871). Décrite en particulier par Abel ef Buck (1967) et par Noakes et Cranwell (1977), la fistulation gastrique n'a guère été employée que pour une approche qualitative des contenus gastriques (Kvasnitsky, 1951 ; Seve et Laplace, 1975). Elle n'a été mise en œuvre pour une étude cinétique de l'évacuation gastrique chez le Porc que très récemment par Low et Pittman (1979).

\section{Matériel et méthodes.}

Six porcs de race Large White mâles castrés d'un poids vif moyen de $56,5 \pm 3,0 \mathrm{~kg}$ ont été utilisés. Sous anesthésie générale à l'halothane, on réalise une laparotomie sur une ligne médio-ventrale, entre l'appendice xyphoïde ef l'ombilic. Une canule en vinyle (diamètres int./ext. 22-30 mm) est mise en place après gastrotomie à mi-hauteur de la grande courbure de l'estomac. Elle est extériorisée, par la plaie opératoire, juste en arrière de l'appendice xyphoïde. Deux de ces porcs ont en outre reçu des électrodes implantées chroniques (Laplace, 1978) sur l'antre gastrique afin d'évaluer l'influence éventuelle de la présence de la fistule gastrique d'une part et des manœurres de collecte d'autre part sur les activités électromyographiques antrales.

Les porcs ainsi préparés sont placés dans des cages à métabolisme et reçoivent exclusivement l'aliment expérimental en un seul repas quotidien, le matin à $9 \mathrm{~h}$. L'aliment utilisé est identique à celui dont l'évacuation gastrique a été précédemment décrite sur la base des collectes au niveau du duodénum (Cuber et Laplace, 1979a) : aliment semi-synthétique comportant 82 p. 100 d'amidon de maïs, 3 p. 100 de cellulose purifiée et 15 p. 100 d'un complément azoté.

Chacun des repas d'épreuve représente l'ingestion de $880 \mathrm{~g}$ de matière sèche (MS), soit $720 \mathrm{~g}$ d'amidon de maïs purifié dont $7 \mathrm{~g}$ d'amidon hydrosoluble, et $61,9 \mathrm{~g}$ de matières azotées $(\mathrm{N} \times 6,25)$ dont $7,1 \mathrm{~g}$ de matières azotées solubles. La quantité correspondante d'aliment frais est distribuée après dilution par 1 I d'eau. Avant le repas d'épreuve $(9 \mathrm{~h})$, on procède au contrôle de la vacuité gastrique : celle-ci est assurée, la canule étant ouverte, par un abreuvement de $500 \mathrm{ml}$. Après ingestion du repas, une seule collecte des contenus gastriques résiduels est effectuée pour chaque jour d'épreuve : l'ouverture de la canule, selon les cas à l'un des temps ci-après : $30 \mathrm{~min}, 1 \mathrm{~h}, 2 \mathrm{~h}, 4 \mathrm{~h}, 7 \mathrm{~h}$ après le repas, autorise la collecte complète des contenus 
gastriques résiduels. Il a été vérifié que celle-ci est effectivement totale, d'une part par rinçage de l'estomac (abreuvement de $500 \mathrm{ml}$ ) et d'autre part par des abattages immédiatement après collecte. Le porc reçoit un repas de remplacement équivalent à la quantité collectée, sur la base de la teneur moyenne en MS des effluents gastriques $(32,17+1,28$ p. 100$)$.

Les contenus gastriques résiduels sont pesés, homogénéisés vigoureusement et échantillonnés. Un premier échantillon d'environ $30 \mathrm{~g}$ est utilisé pour la détermination de la teneur en MS ( $24 \mathrm{~h}$ à l'éfuve à $\left.104^{\circ} \mathrm{C}\right)$. Un second échantillon de même importance est prélevé puis lyophilisé en vue de la détermination de l'azote total (méthode Kjeldahl) et de l'amidon défini pour ce travail comme se référant au glucose et à tous les polymères alpha du glucose (dosage selon Cuber et Laplace (1979b). Un troisième échantillon d'environ $80 \mathrm{~g}$ est soumis à centrifugation $\left(2500 \mathrm{~g}, 20 \mathrm{~min},+4{ }^{\circ} \mathrm{C}\right)$ en vue du dosage de l'azote (Kjeldahl) et des produits d'hydrolyse de l'amidon solubles dans le surnageant de contenus gastriques. Pour le dosage de ces derniers on a recours directement aux étapes enzymatique et colorimétrique de la technique précédemment mise en œuvre (Cuber et Laplace, 1979b), le diméthylsulfoxyde n'étant plus nécessaire pour solubiliser l'amidon.

Ces diverses mesures permettent le calcul de la quantité totale de MS résiduelle dans l'estomac aux temps de collecte, ainsi que des quantités totales de matières azotées $(N \times 6,25)$ et d'amidon correspondantes. Dans le cas des surnageants, la teneur en azote ou amidon solubles est rapportée à la quantité totale d'eau de l'échantillon (calculée sur la base de la teneur en MS) et non au volume du seul surnageant, de façon à calculer les quantités totales de matières azotées et d'amidon solubles.

\section{Résultats.}

1. - Activités électromyographiques antrales. - Aucune anomalie de la fréquence des activités électriques de contrôle de l'antre gastrique n'a été enregistrée chez les porcs fistulés. L'hypermotilité postprandiale, traduite par l'intensification des activités rapides, n'est pas différente de ce qui est observé par ailleurs au laboratoire chez l'animal intact recevant le même régime. L'ouverture de la canule et la vidange rapide des contenus gastriques résiduels conduisent à la disparition des activités rapides, l'électromyogramme antral retournant à l'aspect précédemment observé chez le sujet à jeûn. L'ingestion ultérieure du repas de substitution fait réapparaître les activités rapides témoignant de l'hypermotilité postprandiale. Ces divers aspects sont illustrés par la figure 1.

2. - Quantités résiduelles dans l'estomac. - Les quantités résiduelles dans l'estomac aux 5 temps de la chronologie sont indiquées dans la figure 2, respectivement pour la matière fraîche, la matière sèche, les matières azolées totales et solubles, et l'amidon total et soluble.

L'évacuation gastrique s'avère très progressive. Les quantités résiduelles dans l'estomac sont, en p. 100 de l'ingéré et respectivement à $30 \mathrm{~min}, 1 \mathrm{~h}, 2 \mathrm{~h}, 4 \mathrm{~h}$ et $7 \mathrm{~h}$, de $90,82,66,56$ et 40 p. 100 dans le cas de la matière fraîche, ef de 76, 70, 57, 41 et 26 p. 100 dans le cas de la MS. Cette décroissance de la quantité résiduelle (Q) de matière 

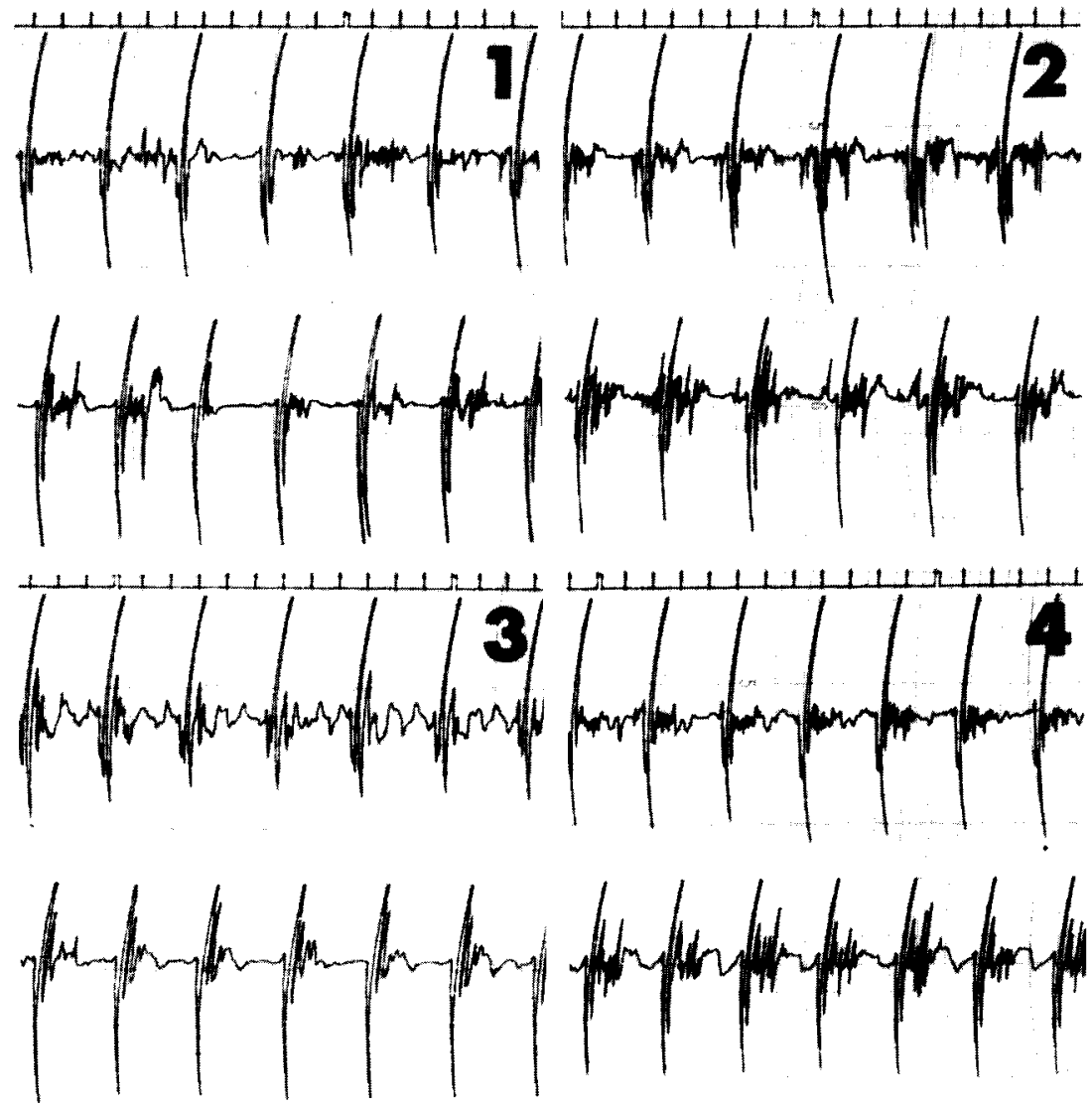

FIG. 1. - Activités électromyographiques de l'antre gastrique chez un porc équipé d'une canule gasirique permanente à mi-hauteur de la grande courbure de l'estomac. Chacun des 4 tracés comporte de bas en haut : la base de temps ( $5 \mathrm{sec}$.) ; l'électromyogramme (EMG) dérivé $9,5 \mathrm{~cm}$ en aval de la canule soit $5,5 \mathrm{~cm}$ en amont du pylore ; l'EMG dérivé $11 \mathrm{~cm}$ en aval de la canule soit $4 \mathrm{~cm}$ en amont du pylore.

En 1 : EMG recueilli chez l'animal à jeûn ; En 2 : EMG enregistré au cours d'un repas ; les activifés rapides apparaissent, préludant à l'hypermotilité postprandiale ; En 3 : Cinq minutes après la vidange rapide de l'estomac par la canule (intervenue 30 min après le repas) les activités rapides ont disparu ; En 4 : Dix minutes après ingestion d'un repas de substitution, l'hypermotilité est réinstallée.

sèche peut être ajustée à un modèle linéaire du type $Q=f(t), \log Q=f(t)$ selon Hunt et Spurrell (1951), ou $\sqrt{\mathrm{Q}}=f(t)$ selon Hopkins (1966) :

$$
\begin{aligned}
& \mathrm{Q}=663,8-1,1 \mathrm{x} \\
& \log Q=2,8578-0,0012 x \\
& r=-0,9367 \quad Q \\
& r=-0,9157 \\
& \mathrm{Q}=880 \mathrm{~g} \mathrm{d̀} \mathrm{t}_{0} \\
& \sqrt{Q}=26,2240-0,0277 \times \\
& r=-0,9412 \\
& \log 880=2,9445 \\
& \sqrt{880}=29,6648
\end{aligned}
$$

La variabilité des quantités résiduelles de $M S$, évaluée chez un même animal entre différentes épreuves de collecte, est très faible au temps $30 \mathrm{~min}$ (coefficient de varia- 

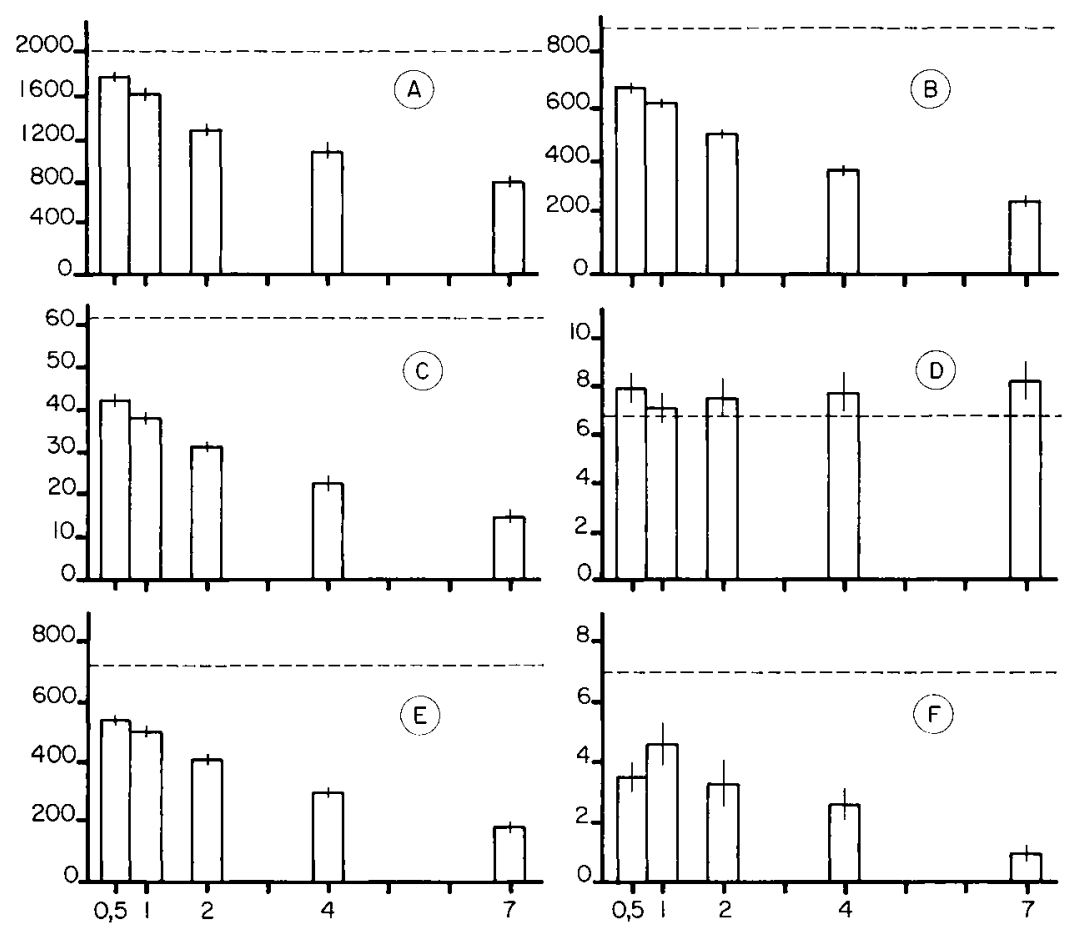

FIG. 2. - Quantités totales moyennes résiduelles dans l'esfomac (exprimées en g), et écarts types de la moyenne, de matière froiche en $A$, de matière sèche en $B$, de matières azotées totales en $C$, de matières azotées solubles en $D$, d'amidon tołal en $E$, ef d'amidon soluble en $F$, ò chacun des temps (en $h$ ) après le repas indiqués en abscisse. Sur chaque graphique, la ligne pointillée correspond à la quantité ingérée. L'amidon est ici défini comme l'ensemble du glucose et de tous ses polymères $\alpha$; les matières azotées sont définies sur la base de l'azote (Kjeldahl) affecté du coefficient 6,25.

tion 0,3 p. 100) ; elle reste modérée (entre 3 et 7 p. 100) jusqu'au stade $4 \mathrm{~h}$ et atteint environ 20 p. 100 au stade $7 \mathrm{~h}$. La variabilité globale sur l'ensemble des quantités résiduelles de MS enregistrées (tous porcs et tous essais confondus) croît progressivement de 4,7 p. 100 au temps $30 \mathrm{~min}$, à 8,7 p. 100 ( $2 \mathrm{~h}$ ), 15,7 p. 100 (4 h) et 28,9 p. 100 (7 h).

La quantité totale de matières azotées résiduelles évolue de façon étroitement corrélée à la quantité de $M S(r=0,936-P<0,01)$. Elle représente un pourcentage pratiquement constant (6,2 à 6,5 p. 100) de la MS aux divers temps de collecte. Mais la proportion de matières azotées solubles dans les matières azotées totales, qui est de 11 p. 100 dans l'aliment, atteint 19 p. 100 dans les contenus gastriques résiduels aux temps $30 \mathrm{~min}$ et $1 \mathrm{~h}$, puis croît progressivement (24, 34 et 55 p. 100 d̀ 2,4 et $7 \mathrm{~h}$ ).

La quantité tolale résiduelle d'amidon est également corrélée de façon étroite à la quantité de $M S(r=0,985-P<0,01)$. Elle représente, selon les temps de collecte 79,4 à 82,3 p. 100 de la MS collectée. La part de l'amidon soluble dans la quantité totale résiduelle d'amidon, de l'ordre de 1 p. 100 dans l'aliment, est comprise entre 0,56 et 0,93 p. 100 selon les temps de collecte. 
3. - Estimation des quantités évacuées par l'estomac. - Les quantités totales de MS, d'amidon, et de matières azotées évacuées par l'estomac à chacun des temps de la cinétique peuvent être estimées par différence $(Q$ ingérée $-Q$ résiduelle). Elles sont exprimées en pourcentages de l'ingéré dans le tableau 1. Dans les conditions de l'expérience, environ un quarł du repas est évacué en $30 \mathrm{~min}$ et les trois quarts de celui-ci en $7 \mathrm{~h}$. Ce mode d'expression (quantité évacuée estimée, en pourcentage de la quantité ingérée) permet de vérifier l'étroite corrélation des quantités de matières azotées, et plus encore d'amidon, aux quantités de matière sèche. Les données antérieurement obtenues par fisfulation duodénale (Cuber et Laplace, 1979b) sont rappelées à titre comparatif dans le tableau 1 . On constate que, au temps $7 \mathrm{~h}$ par exemple, les quantités de MS et d'amidon évacuées par l'estomac, telles qu'elles sont estimées par fistulation gastrique, sont significativement supérieures aux quantités mesurées par fistulation duodénale ( $P<0,005$ pour la $M S$ ef $P<0,001$ pour l'amidon). L'inverse est enregistré pour les matières azotées $(P<0,001)$.

\section{TABLEAU 1}

Evacuation gastrique, au cours d'une période postprandiale de $7 \mathrm{~h}$, de la matière sèche, des matières azotées totales (azote $\succ, 6,25$ ) ef de l'amidon (glucose et tous ses polymères $\alpha$ ), après ingestion de $880 \mathrm{~g}$ de matière sèche d'un régime semi synthéfique à base d'amidon de maïs. Cinétiques comparées selon la technique d'estimation de l'évacuation : F. G. collecte des contenus gastriques résiduels ; F. D. collecte des contenus émis dans le duodénum.

\begin{tabular}{|c|c|c|c|c|c|c|}
\hline \multirow{2}{*}{\multicolumn{2}{|c|}{$\begin{array}{l}\text { Quantité évacuée en p. } 100 \\
\text { de la quantité ingérée }\end{array}$}} & \multicolumn{5}{|c|}{ Temps écoulé depuis l'ingestion du repas } \\
\hline & & $30 \mathrm{~min}$ & $1 \mathrm{~h}$ & $2 \mathrm{~h}$ & $4 \mathrm{~h}$ & $7 \mathrm{~h}$ \\
\hline Matière sèche & $\begin{array}{l}\text { F. G...... } \\
\text { F. D..... }\end{array}$ & $\begin{array}{l}23,9 \\
28,3\end{array}$ & $\begin{array}{l}30,3 \\
38,6\end{array}$ & $\begin{array}{l}42,8 \\
44,8\end{array}$ & $\begin{array}{l}59,1 \\
53,6\end{array}$ & $\begin{array}{l}74,0 \\
64,4\end{array}$ \\
\hline Matières azotée & $\begin{array}{l}\text { S F. G..... } \\
\text { F. D.... }\end{array}$ & $\begin{array}{l}31,3 \\
34,6\end{array}$ & $\begin{array}{l}38,4 \\
47,3\end{array}$ & $\begin{array}{l}48,9 \\
57,8\end{array}$ & $\begin{array}{l}63,0 \\
81,3\end{array}$ & $\begin{array}{l}75,9 \\
98,7\end{array}$ \\
\hline Amidon & $\begin{array}{l}\text { F. G.... } \\
\text { F. D..... }\end{array}$ & $\begin{array}{l}25,3 \\
26,4\end{array}$ & $\begin{array}{l}31,0 \\
35,6\end{array}$ & $\begin{array}{l}42,9 \\
40,3\end{array}$ & $\begin{array}{l}58,9 \\
46,4\end{array}$ & $\begin{array}{l}74,7 \\
53,9\end{array}$ \\
\hline
\end{tabular}

\section{Discussion.}

1. - Evaluation de l'usage de la fistulation gastrique.

Le choix de la fistulation gastrique, donc de la mesure des quantités résiduelles dans l'estomac, pour une étude de l'évacuation gastrique présente deux inconvénients. Le calcul par différence des quantités évacuées à partir des quantités résiduelles mesurées, suppose une absorption gastrique nulle pour être rigoureusement exact. Le second inconvénient est lié au fait que la cinétique d'évacuation gastrique est reconstituée à partir de données ponctuelles et non le résultat d'une mesure continue à la suite d'un même repas. II s'agit là cependant d'une technique classique, dite de repas d'épreuve, largement utilisée chez l'Homme depuis Hunt (1949).

En dépit de ces inconvénients, l'usage de la fistulation gastrique présente divers avantages. Son utilisation est beaucoup moins contraignante pour l'expérimentateur. 
Son innocuité paraît totale, au moins au plan des activités électromyographiques de l'antre, ce qui n'est pas le cas pour une fistulation duodénale réentrante (Laplace, 1979). La longévité expérimentale de la préparation n'est pas un facteur limitant comme ce peut être le cas après fistulation réentrante du duodénum. En plus de ces avantages pratiques, la fistulation gastrique permet aussi de s'affranchir de l'interférence quantitative des sécrétions biliaire et pancréatique, habituellement drainées avec les digesta par une fistule duodénale. Enfin, elle autorise un contrôle de la vacuité gastrique préalable à l'ingestion du repas d'épreuve, éliminant ainsi l'interférence possible des reliquats du repas antérieur (Cuber et Laplace, 1979a).

Dans nos conditions expérimentales, l'estomac s'est avéré vide lors du contrôle préalable à l'expérience, la quantité de MS résiduelle récupérée par le rinçage étant négligeable. On notera au demeurant que selon le modèle linéaire qui fournit la meilleure corrélation négative (racine carrée de la quantité résiduelle $-\mathrm{g}$ - en fonction du temps - min) la vacuité gastrique totale devrait être atteinte en $15 \mathrm{~h}$ $47 \mathrm{~min}$. Cette extrapolation est compatible avec les données de l'expérience ce qui n'est pas le cas pour les deux autres modèles linéaires. A l'autre extrême, l'extrapolation au temps zéro ne permet pas, pour aucun des 3 modèles, de recouvrer une valeur égale à l'ingéré. Cela est à relier au fait que l'évacuation gastrique débute pendant l'ingestion du repas d'épreuve (Laplace ef Tomassone, 1970) el que, en conséquence, l'estomac ne contient jamais l'intégralité du repas d'épreuve.

Cela étant, la distribution d'un seul repas par jour, limité à $880 \mathrm{~g}$ de MS pour faciliter la comparaison aux résultats antérieurs (Cuber et Laplace, 1979a et b), permet seulement aux animaux de maintenir leur poids vif. La possibilité d'assurer, par la fistule gastrique, la vacuité de l'estomac avant ingestion du repas d'épreuve devrait done dans l'avenir permettre de s'affranchir de telles contraintes. Enfin, la distribution à l'animal, après le prélèvement des contenus gastriques, d'un repas de substitution quantitativement équivalent ne semble pas perturbatrice. Cette méthodologie n'a pas été comparée à la restitution des digesta du repas d'épreuve après échantillonnage, pratiquée par Low et Pittman (1979).

\section{2. - Cinétiques comparées d'évacuation gastrique selon le mode de collecte.}

La cinétique d'évacuation gastrique de la MS, éłablie par le calcul sur la base des quantités résiduelles dans l'estomac, diffère sensiblement (voir tabl. 1) de celle qui a été enregistrée pour le même aliment par collecte au niveau d'une fistule duodénale proximale (Cuber et Laplace, 1979a). Le rythme d'évacuation de l'estomac, évalué par collecte des contenus gastriques, s'avère plus lent pendant les deux premières heures, et à l'inverse plus rapide au-delà. L'absence d'une pression endoluminale normale dans l'intestin grêle lorsque les canules duodénales sont ouvertes est susceptible de modifier le rythme de l'évacuation gastrique (Low et Zebrowska, 1977). Cependant, les observations de ces auteurs ne permettent pas d'expliquer la différence observée dans l'évacuation d'un même régime, mesurée au niveau d'une fistule duodénale (Cuber et Laplace, 1979a) ou par collecte des contenus gastriques résiduels. L'usage de marqueurs dans l'une ou l'autre des expérimentations n'aurait pas permis d'accorder plus de crédit aux informations recueillies pour choisir entre les deux techniques. En effet, Holmes, Bayley et Horney (1974) constatent chez des 
porcs, lors de collecte au niveau d'une fistule duodénale réentrante, la séparation du marqueur et de la matière sèche ef la fixation du marqueur sur la muqueuse gastrique. En dépit des critiques formulées à l'encontre des collectes au niveau d'une fistule duodénale réentrante chez les ruminants (Faichney, 1974 ; Mac Rae, 1974), les aléas inhérents à l'usage de marqueurs (manque d'homogénéité de distribution, association variable à la matière sèche, taux de récupération irrégulier) sont trop importants pour offrir quelque intérêt chez nos porcs accoutumés à des collectes quotidiennes qui sont par ailleurs rigoureusement totales.

En réalité le rythme de l'évacuation gastrique de la $M S$, enregistrée au niveau d'une fistule duodénale, conduit à laisser subsister dans l'estomac après $24 \mathrm{~h}$ un reliquat de MS plus important que lors de fistulation gastrique. L'interférence de ce reliquat, qui ne peut être éliminé avant l'épreuve, est susceptible de rendre compte pour partie du surcroît d'évacuation précoce enregistrée au niveau d'une fistule duodénale (Cuber et Laplace, 1979a) par rapport aux données fournies par collecte des contenus gastriques résiduels. Il s'ajoute aux digesta collectés par fistulation duodénale, la MS des sécrétions biliaire et pancréatique. Le cumul de ces deux ordres d'effets peut expliquer les quelque $40 \mathrm{~g}$ de MS qui séparent les résultats obtenus par les 2 techniques au temps $30 \mathrm{~min}$. Enfin, il n'est pas exclu que la différence des deux cinétiques d'évacuation de la MS résulte également, en dépit des précautions, d'une légère imperfection de la restitution des contenus lors de fistulation duodénale (Low, 1979).

En ce qui concerne l'évacuation des matières azotées, l'interférence de l'apport endogène, lié aux sécrétions drainées avec les digesta par une fistulation duodénale, rend comple de l'écart important des cinétiques établies par chacune des deux techniques (voir tabl. 1). Alors que, $7 \mathrm{~h}$ après un repas de $880 \mathrm{~g}$ de $\mathrm{MS}$, l'évacuation des matières azotées n'est plus corrélée à celle de la MS lors de collecte par voie duodénale (Cuber et Laplace, 1979b), la corrélation subsiste $(r=0,5608-p<0,05)$ dans le cas de collecte par une fistule gastrique, ce qui correspond à la stabilité du pourcentage de matières azotées totales dans la MS. La croissance du pourcentage de matières azotées solubles dans les matières azotées totales des contenus gastriques résiduels pourrait refléter à la fois l'addition d'une fraction directement soluble (pepsine gastrique, urée salivaire) de l'apport endogène, et une certaine hydrolyse des matières azotées alimentaires. Les résultats disponibles ne permettent pas de dissocier ces deux composantes possibles.

L'évacuation gastrique de l'amidon, estimée sur la base des contenus gastriques résiduels, s'avère suivre très étroitement celle de la MS à tous les temps de la cinétique, contrairement à ce qui a été enregistré par fistulation duodénale (Cuber et Laplace, $1979 b)$. Cette différence équivaut, lors de collecte des contenus gastriques résiduels, à une évacuation supplémentaire apparente en $7 \mathrm{~h}$ de 20 p. 100 de l'amidon ingéré. Si importante que puisse être la production par fermentation d'acides organiques dans l'estomac (Friend, Cunningham et Nicholson, 1963 ; Argenzio et Southworth, 1975 ; Clemens, Stevens et Southworth, 1975), elle ne saurait en aucune façon expliquer la différence observée entre les résultats obtenus par les deux techniques de collecte. On sait par ailleurs que la digestibilité apparente de la matière sèche n'est guère que de 4 p. 100 au niveau gastro-duodénal (Keys et De Barthe, 1974), ef que si l'amidon proprement dit est déjà attaqué, sa dégradation n'est pas suffisamment avancée pour qu'une digestion appréciable soit enregistrée à ce niveau. On notera aussi l'analogie 
entre l'écart des quantités d'amidon apparemment évacuées en $7 \mathrm{~h}$, selon l'une ou l'autre technique - écart de 20 p. 100 alors que les trois quarts du repas ont été évacués - ef les résultats de Sambrook (1979). Cet auteur observe en effet que, dans des bilans de 24 h, 20 à 50 p. 100 du glucose issu de l'hydrolyse acide des contenus digestifs ne sont pas récupérés au niveau d'une canule duodénale. Ce défaut ne peut en aucune façon être attribué à une quelconque absorption à ce niveau très proximal (15 cm seulement du pylore) et compte tenu de ce qui a été dit précédemment.

II apparaît donc que l'évacuation gastrique de l'amidon est sous-estimée lors de collecte des digesta au niveau d'une fistule duodénale. La proposition inverse (surestimation par collecte des contenus gastriques résiduels) n'est en effet pas plausible compte tenu du fait que : 1) la teneur en amidon de la MS reste stable (81 p. 100 dans l'aliment et 79,4 à 82,3 p. 100 dans les contenus gastriques aux divers temps de collecte) ; 2) la proportion de l'amidon soluble dans l'amidon total des contenus gastriques résiduels reste toujours en deça de sa proportion dans l'aliment. L'origine de cette sous-estimation de l'amidon présent dans les contenus duodénaux pourrait être recherchée dans une interférence des pigments biliaires lors du dosage du glucose vrai par la glucose oxydase et la peroxydase, aussi bien dans le précédent travail (Cuber et Laplace, 1979b) que dans celui de Sambrook (1979). Cette hypothèse est étayée par la démonstration (Witte, Brown et Feld, 1978) de l'interférence de la bilirubine sur la détection de $\mathrm{H}_{2} \mathrm{O}_{2}$ dans divers dosages fondés sur l'utilisation de la peroxydase.

\section{3. - Relations entre évacuation gastrique et absorption des glucides.}

La très probable réalité de cette sous-estimation des quantités d'amidon présentes dans les contenus duodénaux oblige à réviser l'interprétation préalablement avancée (Cuber et Laplace, 1979b ; Rérat, 1980) du rôle de l'évacuation gastrique à l'égard de

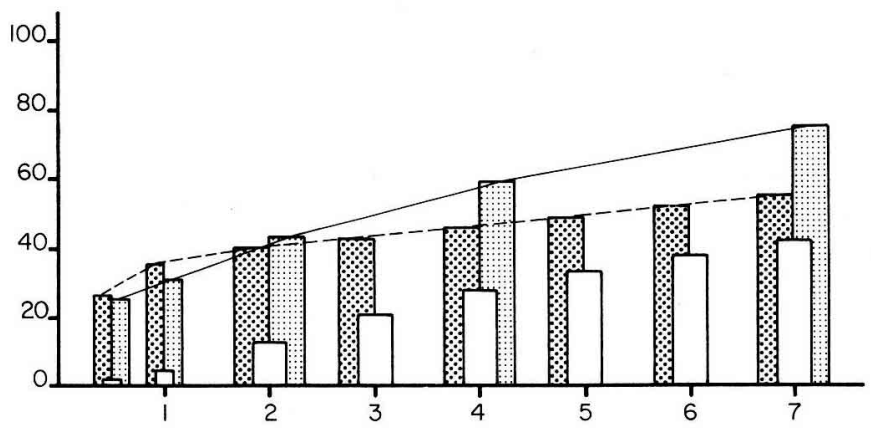

FIG. 3. - Cinétiques comparées, au cours d'une période de $7 \mathrm{~h}$ oprès ingestion d'un régime semi-synthétique à base d'amidon de maïs, de l'évacuation de l'amidon total (défini comme pour la fig. 2) sur la base de la collecte des digesta déversés dans le duodénum (pointillé, à gauche) ou sur la base d'une estimation ò partir des contenus gastriques résiduels (pointillé, à droite) ef de l'absorption des sucres réducteurs (colonnes blanches). Les quantités évacuées, appréciées par l'une ou l'autre fechnique, et les quantités absorbées, sont toutes exprimées en p. 100 de la quantité ingérée.

Les données relatives à l'absorption des sucres réducteurs sont celles de Rérat (1980). Les résultats des collectes duodénales sont ceux de Cuber et Laplace (1979b). 
l'absorption des produits d'hydrolyse de l'amidon. La confrontation des cinétiques d'évacuation gastrique de l'amidon, mesurée par différence (collecte des contenus gastriques résiduels) ou directement (collecte duodénale) et exprimée dans les deux cas en pourcentage de l'amidon ingéré, avec l'évolution du coefficient d'absorption (absorbé/ingéré, p. 100) des sucres réducteurs tolaux issus de la digestion de l'amidon (Rérat, 1980), est illustrée dans la figure 3. Au-delà des premières heures après le repas, une similitude croissante de la cinétique d'évacuation gastrique établie sur la base des collectes duodénales et de la cinétique d'absorption est observée. Leur décalage initial avait été imputé au temps nécessaire à la progression des digesta dans l'intestin grêle ef à la mise en jeu progressive des surfaces absorbantes (Cuber et Laplace, 1979b). Par contre, il existe un retard constant et considérable de l'absorption des sucres réducteurs par rapport à l'évacuation gastrique de l'amidon estimée sur la base des collectes de contenus gastriques résiduels. Ce décalage représente environ 30 p. 100 de l'ingéré, tout au long de la période de $7 \mathrm{~h}$. Sept heures après le repas, la part de l'ingéré effectivement absorbée n'équivaut qu'à environ la moitié de la fraction de l'ingéré qui a été évacuée par l'estomac.

En somme, l'analyse de l'évacuation gastrique fondée sur la collecte des contenus gastriques résiduels conduit à rejeter la conception précédemment refenue (Cuber et Laplace, 1979b) selon laquelle l'évacuation gastrique serait le facteur limitan' contrôlant la cinétique d'apparition des sucres réducteurs dans la veine porte. L'absorption elle-même n'est pas non plus le facteur limitant, lors d'ingestion d'amidon de maïs, puisque le coefficient d'absorption reste constant lors d'augmentation de la quantité ingérée (Rérat, 1975). Ce double constat conduit donc à rechercher l'origine du décalage soit dans les processus d'hydrolyse de l'amidon soit dans l'importance de la métabolisation par la paroi. En ce qui concerne cette dernière hypothèse, aucune information quantitative n'est disponible pour évaluer la proportion du glucose prélevé ef utilisé pour son propre compte par la paroi intestinale. On notera de surcroît que l'écart enregistré entre quantités évacuées et quantités absorbées est sous estimé puisque le glucose vrai ne représente que 88,5 à 90,6 p. 100 des sucres réducteurs apparaissant dans le sang de la veine porte au cours des 8 heures conséculives à l'ingestion d'amidon de maïs (Rérat, 1977). En ce qui concerne les processus d'hydrolyse de l'amidon, l' $\alpha$-amylase pancréatique semble largement surabondante, puisqu'une privation à court terme de la sécrétion pancréatique ne réduit guère que de 20 p. 100 environ l'absorption apparente des sucres réducteurs (Rérat et al., 1977). En l'absence de défaut quantitatif d'amylase pancréatique, seule la vitesse d'hydrolyse des chaînes d'amidon par l'amylase serait limiłante. L'étape ultime (hydrolyse des oligosaccharides) ne peut en effet être suspectée de freiner l'hydrolyse de l'amidon puisqu'il n'y a jamais que de très faibles quantités de sucres hydrosolubles dans les contenus digestifs (Sambrook, 1979). Pourtant on admet habituellement que l'hydrolyse in vitro de l'amidon de maïs par l' $\alpha$-amylase est relativement rapide; comparativement à d'autres amidons de céréales ou de tubercules. Mais, là encore, on ne dispose d'aucune information relative à la vitesse d'hydrolyse in vivo des chaînes d'amidon.

En conclusion : La fistulation chronique de l'estomac constitue une technique particulièrement intéressante pour l'étude de la cinétique d'évacuation gastrique. D'une 
totale innocuité, elle offre maints avantages tels que possibilité de contrôle préalable de la vacuité gastrique, non interférence quantitative des sécrétions biliaire et pancréatique, absence d'interférence qualitative des pigments biliaires dans le dosage de l'amidon. Son usage permet de constater que l'évacuation gastrique, plus importante en $7 \mathrm{~h}$ que ne le montre une collecte duodénale, n'est pas réellement le facteur limitant de l'absorption des produits d'hydrolyse de l'amidon.

\begin{abstract}
Journées Ingestion-Digestion-Absorption de l'Association française de Nutrition, Paris, 15-16 novembre 1979.
\end{abstract}

\title{
Références
}

ABEL M., BUCK W. B., 1967. A technique for gastric cannulation of swine. Cornell Vef., 57, 383-389. ARGENZIO R. A., SOUTHWORTH M., 1975. Sites of organic acid production and absorption in gastro intestinal tract of the pig. Am. J. Physiol., 228, 454-460.

CLEMENS E. T., STEVENS C. E., SOUTHWORTH M., 1975. Sites of organic acid production and pattern of digesta movement in the gastro intestinal tract of swine. J. Nutr., 105, 759-768.

COLIN G., 1871. Traité de physiologie comparée des animaux. T. 1, 2e éd. Baillière ed., Paris.

CUBER J. C., LAPLACE J. P., 1979a. Evacuation gastrique de la matière sèche d'un régime semi-purifié à base d'amidon de maïs chez le porc. Ann. Biol. anim. Bioch. Biophys., 19, 899-905.

CUBER J. C., LAPLACE J. P., 1979b. Evacuation gastrique de l'amidon el de l'azote d'un régime semipurifié à base d'amidon de maïs chez le porc. Ann. Zoofech., 28, 173-184.

FAICHNEY G. J., 1974. The use of markers to partition digestion within the gastro intestinal tract of ruminants, 277-291. In McDonald I. W., Warner A. C. I., Digestion and melabolism in the ruminant, Univ. New-England Publ. Unit., Armidale, Australia.

FRIEND D. W., CUNNINGHAM H. M., NICHOLSON J. W. G., 1963. The production of organic acids in the pigs. 2) The effect of diet on the levels of volatile fatty acids and lactic acid in sections of the alimentary tract. Canad. J. Anim. Sci., 43, 156-168.

HOLMES J. H. G., BAYLEY H. S., HORNEY F. D., 1974. Digestion of dry and high-moisture maize diets in the stomach of the pig. Br. J. Nutr., 32, 639-646.

HOPKINS A., 1966. The pattern of gastric emptying. A new view of old results. J. Physiol. London, 182, 144-149.

HUNT J. N., 1949. The simultaneous estimation of the absorption of water sulphaguanidine from the stomach of man. J. Physiol. London, 109, 134-141.

HUNT J. N., SPURRELL W. R., 1951. The pattern of emptying of the human stomach. J. Physiol. London, 113, 157-168.

KEYS J. E., DE BARTHE J. V., 1974. Site and extent of carbohydrates, dry matter, energy and protein digestion and the rate of passage of grain diets in swine. J. Anim. Sci., 39, 57-62.

KVASNITSKY A. V., 1951. Voprosy fiziologii prischevarenija u svinej, Moscow : Sel' Khozgiz (traduit par D. E. Kidder).

LAPLACE J. P., 1978. Organisation de la motricité de l'intestin grêle chez le Porc et influence de l'alimentation. Ann. Zootech., 27, 377-408.

LAPLACE J. P., 1979. Stomach and small intestine motility in the pig. Electromyography in nutritional studies. In Seminar on digestion and absorption in cannulated pigs, Shinfield-U. K. (in press).

LAPLACE J. P., TOMASSONE R., 1970. Evacuation gastro-duodénale chez le porc. Fistulation chronique par voie thoracique extrapleurale ; recherche d'une technique d'analyse mathématique de l'évacuation. Ann. Zootech., 19, 303-332.

LOW A. G., 1979. Observations on the objectives and methodology of research on digestion and absorption in pigs. In Seminar on digestion and absorption in cannulated pigs, Shinfield-U. K. (in press).

LOW A. G., PITTMAN R. J., 1979. Dietary factors influencing gastric emptying in growing pigs. 30th Annu. Meet. europ. Assoc. Anim. Prod., Harrogate-U. K.

LOW A. G., ZEBROWSKA T., 1977. Dry matter and nitrogen in the duodenal contents of growing pigs : a discrepancy explained. Br. J. Nutr., 38, 145-147. 
MACRAE J. C., 1974. The use of re-entrant cannulae to partition digestive function within the gastro intestinal tract of ruminants. 261-276. In McDonald I. W., Warner A. C. I., Digestion and metabolism in the ruminant, Univ. New-England Publ. Unit, Armidale, Australia.

NOAKES D. E., CRANWELL P. D., 1977. Some experimental surgical techniques on the alimentary tract of young pigs. Res. vet. Sci., 22, 243-250.

RÉRAT A., 1975. Mesure quantitative in vivo de l'absorption chez le porc. Application aux sucres et aux acides aminés. In Réanimation entérale à faible débit continu, Coll. INSERM, 53, 47-62.

RÉRAT A., 1977. Mise au point d'une méthode quantitative d'étude de l'absorption digestive chez le porc : applications à quelques problèmes nutritionnels concrets. Bull. Acad. vet. de France, 50, 93-107.

RÉRAT A., 1980. Digestion and absorption of nutrients in the pig. Some new data concerning protein and carbohydrates. Symp. Future perspectives in pig nutrition, Reading-U. K., déc. 1979. Proc. Nuir. Soc. (in press).

RÉRAT A., CORRING T., VAISSADE P., VAUGELADE P., 1977. Postprandial absorption of $\alpha$-aminonitrogen and sugars in pigs. Quantitative measurement using discontinuous pancreatic derivation. Ann. Biol. anim. Bioch. Biophys., 17, 583-588.

RÉRAT A., LOUGNON J., 1963. Ełudes sur le transit digestif chez le Porc. Ann. Biol. anim. Bioch. Biophys., 3 ( $n^{\circ}$ Hors Série), 21-27.

SAMBROOK I. E., 1979. Studies on digestion and absorption in the intestines of growing pigs. 7. - Measurements of the flow of total carbohydrate, total reducing substances and glucose. Br. J. Nutr., 42, 267-277.

SEVE B., LAPLACE J. P., 1975. Influence de la substitution des protéines du lait par des protéines de poisson sur quelques caractéristiques du contenu gastrique chez le porcelet sevré à 12 jours. Ann. Zootech., 24, 43-57.

WITTE D. L., BROWN L. F., FELD R. D., 1978. Effects of bilirubin on detection of hydrogen peroxide by use of peroxidase. Clin. Chem., 24, 1778-1782. 